\title{
A Study on the Heterogeneity of the Light-Harvesting Complex II from Ectothiorhodospira sp. after Acid/Chaotropic Treatment
}

\author{
André Buche* and Rafael Picorel \\ Estación Experimental de Aula Dei (CSIC), Apartado 202, E-50080 Zaragoza, Spain. \\ Fax:34-976-575620. E-mail: abuche@eead.csic.es \\ * Author for correspondence and reprint requests \\ Z. Naturforsch. 55c, 943-947 (2000); received July 19/August 24, 2000 \\ Light-Harvesting Complex, Ectothiorhodospira, Bacteriochlorophyll
}

The light-harvesting complex II of the purple bacteria has two strong near infrared electronic absorption bands, around $800(\mathrm{~B} 800)$ and $850(\mathrm{~B} 850) \mathrm{nm}$, arising from the $Q_{v}$ transitions of bacteriochlorophyll $a$. It was previously reported that under some specific acid/ chaotropic conditions the B850 bacteriochlorophylls of the light-harvesting complex II of Ectothiorhodospira sp. are strongly reorganised. Part of these pigments absorbs at $843 \mathrm{~nm}$ while another set absorbs around $858 \mathrm{~nm}$. The current work should investigate whether a mix of two different complexes could generate the 843- and 858-nm bands. Acid/chaotropic conditions inducing the reorganisation of B850 were reproduced on a sample bound to an ionic-exchange column. The chromatographic pattern was found strongly homogeneous. The findings indicate that the heterogeneity of the reorganised B850 results from two forms of differently structured bacteriochlorophylls bound to the same polypeptide backbone.

\section{Introduction}

Three different types of antenna complexes can be isolated from photosynthetic purple bacteria. The LHI or B880 (Cogdell, 1986; Picorel and Gingras, 1988; Sundström and van Grondelle, 1996) is present in all purple bacteria and is intimately associated with the reaction center. The LHII or B800-850 (Feick and Drews, 1978; Clayton and Clayton, 1981; Doi et al., 1991; Walker et al., 1991) and LHIII or B800-820 (Hayashi and Morita, 1980; Cogdell et al., 1983) are arranged more peripherally (Monger and Parson, 1977). Their concentration in the cells can vary with growth conditions. All of these antenna complexes are spectrally characterised by one or two strong near infrared (NIR) electronic absorption bands arising from the $Q_{y}$ transition of the bacteriochlorophyll (BChl) $a$.

The crystal structure of the LHII complex from Rhodopseudomonas (Rps.) acidophila (McDermott et al., 1995) and Rhodospirillum (Rs.) molischianum (Koepke et al., 1996) were established.

Abbreviations: BChl, bacteriochlorophyll; BPheo, bacteriopheophytin; LH, light-harvesting; B800, 800-nm absorption band; B850, 850-nm absorption band; LDAO, lauryldimethylamine $\mathrm{N}$-oxide.
The active assembly consists of two concentric cylinders of $9 \alpha / 9 \beta$ helical protein subunits in Rps. acidophila or $8 \alpha / 8 \beta$ in Rs. molischianum that hold the pigment molecules. Eighteen or sixteen B850 BChls (depending on the species) are sandwiched between the protein helices near the periplasmic side of the cytoplasmic membrane and other set of nine or eight B800 BChls (depending on the species) are positioned towards the cytoplasmic side. Two B850 BChls are located at a typical center-to-center distance of about $1 \mathrm{~nm}$, two B800 BChls at about $2.1 \mathrm{~nm}$, and the two rings are about $1.8 \mathrm{~nm}$ apart (McDermott et al., 1995).

Recently, we studied the effect of acid $\mathrm{pH}$, both in the absence or presence of urea on the LHII complex from Ectothiorhodospira sp. (Buche et al., 2000; Buche, 2000). Around pH 5.5-6.0 B850 was specifically and reversibly affected by a blue shift suggesting that a histidine residue most probably participated in the in vivo shifting of the absorption in the red. This transition was effective with or without urea present. Under strong chaotropic conditions, a second transition occurred around $\mathrm{pH} 2.0$, affecting the B800 band irreversibly and B850 reversibly. Under these conditions a blue shift from 856 to $842 \mathrm{~nm}$ occurred and a new and strong circular dichroism signal from the new 842$\mathrm{nm}$ band was observed. Reversion to the original
This work has been digitalized and published in 2013 by Verlag Zeitschrif für Naturforschung in cooperation with the Max Planck Society for the Advancement of Science under a Creative Commons Attribution-NoDerivs 3.0 Germany License. 
experimental conditions induced a red shift up to $856 \mathrm{~nm}$ of the B850 band but the circular dichroism signal remained mostly unaffected. Furthermore, gaussian deconvolution and second derivative of the reverted spectra at $\mathrm{pH} 8.0$ after strong acid treatment indicated that the new B850 band was actually composed of two bands centred at 843 and $858 \mathrm{~nm}$. We ascribed the $858-\mathrm{nm}$ band to BChls that underwent a reversible spectral shift, and that the $843-\mathrm{nm}$ band is due to oligomeric bacteriopheophytin (Bpheo) formed from a part of the B850 BChls. This new oligomer should be responsible for the observed strong and mostly conservative circular dichroism signal.

The current work was aimed to investigate the possibility that the reverted form could be a mix of both native and reorganised complexes. The results indicate that the $\mathrm{B} 850 \mathrm{BChls}$ of the reverted complex are fixed to the same polypeptide backbone.

\section{Materials and Methods}

\section{Preparation of the LHII complex}

The photosynthetic bacterium Ectothiorhodospira sp. was grown photosynthetically as described by Lefebvre et al. (1984). Preparations of the LHII antenna were obtained as described previously (Ortiz de Zarate and Picorel, 1994) with some modifications (Buche and Picorel, 1998).

\section{Sample treatment}

One $\mathrm{ml}$ of LHII complex at a concentration corresponding to 4 absorption units measured at $795 \mathrm{~nm}$ under a $1 \mathrm{~cm}$ pathlength was loaded onto a Fractogel TSK-DEAE 650 s column of $5 \times 1 \mathrm{~cm}$ equilibrated with $50 \mathrm{~mm}$ phosphate buffer, $\mathrm{pH} 8.0$ and including $0.05 \%$ (wt/vol) lauryldimethylamine $\mathrm{N}$-oxide (LDAO). The sample was then washed with $45 \mathrm{ml}$ phosphate buffer $\mathrm{pH} 2$ and 8.9 м urea. The column was successively washed by phosphate buffer pH 8 and, phosphate buffer, pH 8/400 mm $\mathrm{NaCl}$. The sample was then eluted with phosphate buffer $400 \mathrm{~mm} \mathrm{NaCl}$ and $0.2 \%$ LDAO.

\section{Spectroscopic measurements}

Absorption spectra were measured in a Beckman DU-640 spectrophotometer. Circular dichroism spectra were scanned in a Jasco spectropolaro- graph (model 720) using a 1-mm optical path cell. Spectra of dialysed samples were normalized for volume modification. The reproducibility of the results was verified at least 5 times on different preparations of the LHII complex. Spectral gaussian deconvolution was carried out using the GRAMS software (Galactic Industries Co., Salem $\mathrm{NH}$ ).

\section{Pigment extraction}

Pigments were extracted with an acetone/methanol $(7 / 2, \mathrm{vol} / \mathrm{vol})$ mixture. After sonication for $1 \mathrm{~min}$, the extract was centrifuged in a microfuge for $2 \mathrm{~min}$ to pellet the unsolved material and the supernatant recovered for further analyses. When necessary the $\mathrm{BChl}$ was converted to $\mathrm{BPheo}$ by adding a few $\mu \mathrm{l}$ of concentrated $\mathrm{HCl}$ to the pigment extract.

\section{Results}

As mentioned in the Introduction the 858-nm band of the reorganized LHII complex was ascribed to $\mathrm{BChl}$ and the $843 \mathrm{~nm}$ band to oligomeric BPheo (Buche et al., 2000). The absorption spectra of pigments extracted in organic solvent (see Materials and Methods) both from reverted and native LHII complexes are compared (Fig. $1 \mathrm{~A}$ and B). Acidification of these pigments induces a total pheophytinisation of BChl. The band at $525 \mathrm{~nm}$ is characteristic of this process concomitantly with an increase of the Soret band intensity and a blueshift/hypochromism of the $Q y$ band. The difference between the spectra before and after acidification presented in Fig. 1C is less important in case of the reverted sample, precisely because of the presence of BPheo in this complex. From this figure and considering the modification of intensity of the Soret or the $Q y$ band it could be deduced that $\mathrm{BPheo}$ in the reverted complex represents around $30-35 \%$ of the initial BChls.

The effect of the presence of BPheo on the heterogeneity of the reorganized complex was tested subjecting a native sample to acid/chaotropic conditions after previous fixation to the same ionic liquid chromatography column used to purify the LHII complex (see Materials and Methods). Reversion from acid/chaotropic to standard buffer at $\mathrm{pH} 8$ was also achieved on the column. The elution profile of the reverted sample is pre- 

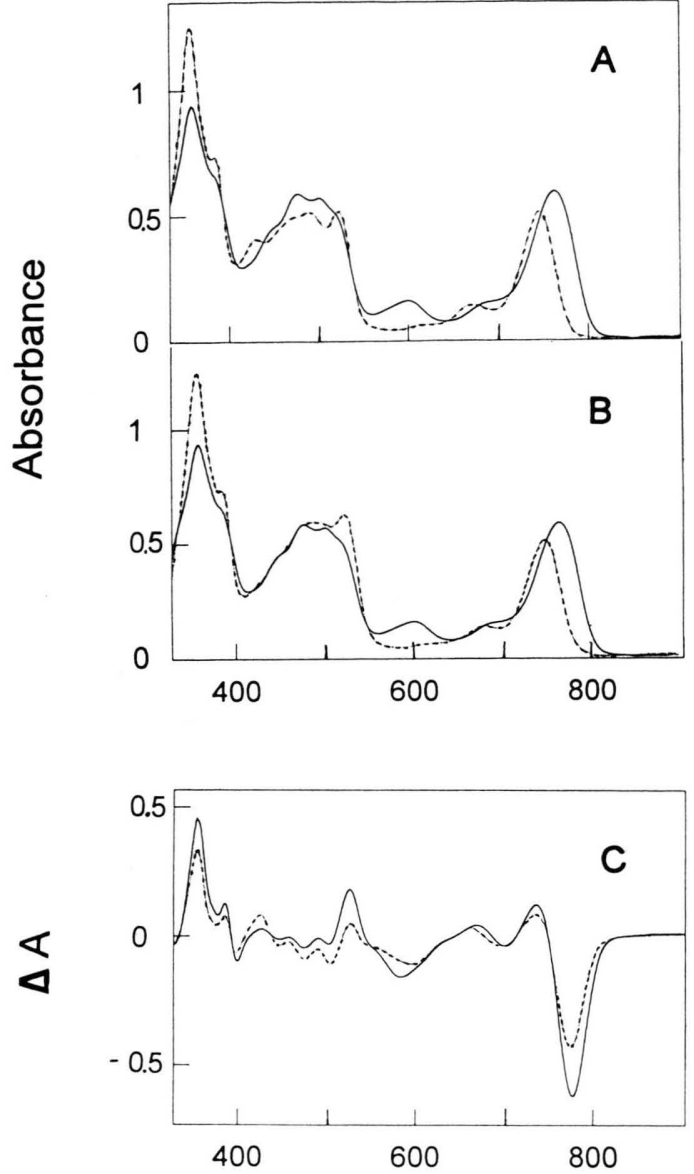

Wavelength $(\mathrm{nm})$

Fig. 1. Organic extraction of pigments from reorganised (A) and native (B) LHII complexes before (solid line) and after acidification (dotted line) (see Materials and Methods).

Difference between the absorption spectra of pigments before and after acidification (C). Both pigments from reorganised (dotted line) and native (solid line) complexes are presented.

sented by Fig. 2. Elution of the complex occurs uniformly. The sample corresponding to the maximum of elution presents similar near infrared spectroscopic properties as that of the reverted sample directly submitted to acid/chaotropic conditions (not shown). Figure 3 compares $Q x$, carotenoid and the Soret bands of reverted samples (directly treated by acid/chaotropic conditions or previously fixed to the column) with the native sample before treatment. Both reverted samples

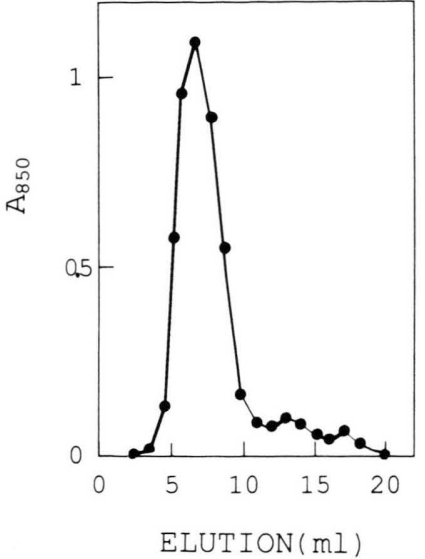

Fig. 2. Elution profile of LHII complex previously loaded onto a Fractogel TSK-DEAE 650s column, treated by acid/chaotropic conditions washed and eluted (see Materials and Methods).

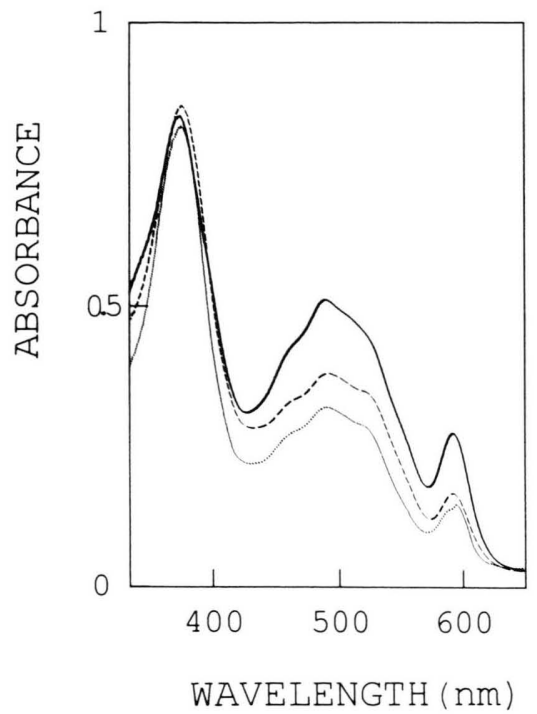

Fig. 3. UV-VIS spectra of the native complex (solid line), the sample corresponding to the maximum of elution of Fig. 2 (dotted line) and a sample submitted $45 \mathrm{~min}$ to acid/chaotropic treatment (dashed line). Spectra were normalised on B850.

are similarly affected by a loss of the carotenoid and the $Q x$ bands. The Soret band is poorly affected but a definitive conclusion about this band is difficult because of the presence of different contributions in this wavelength region: $\mathrm{BChl}$, BPheo but also the $\left({ }^{1} \mathrm{C} \rightarrow{ }^{1} \mathrm{~A}\right)$ transition of the carotenoid (Buche and Picorel, 2000). The absorp- 
tion spectra of the samples constituing the main peak of elution are similar to that of the maximum of elution (not shown). Some differences could be found in the last samples of this peak. For example, Fig. 4 compares the sample corresponding to the maximum of elution to the samples after 9 and $10 \mathrm{ml}$ of elution. This heterogeneity does not relate to the position of B850 which stays centered around $853 \mathrm{~nm}$ but is rather due to the $\mathrm{B} 850 / \mathrm{B} 800$ ratio, the presence of monomeric $\mathrm{BChl}$ and the presence of a band around $360 \mathrm{~nm}$ which could correspond to the intensification of the $\left({ }^{1} \mathrm{C} \rightarrow{ }^{1} \mathrm{~A}\right)$ transition of the carotenoid (Buche and Picorel, 2000). The chromatographic analysis reveals that the great majority of the reverted complex is homogeneous.

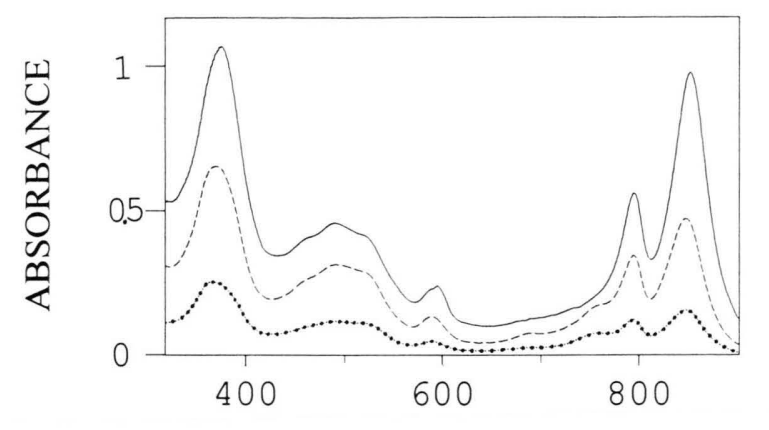

WAVELENGTH (nm)

Fig. 4. Comparison of absorption spectra corresponding to different steps of the elution profile: the maximum of elution (solid line), after 9 (dashed line) and 10 (dotted line) $\mathrm{ml}$ of elution.

\section{Discussion}

The current work was aimed to investigate the possibility that the heterogeneity of B850 induced by the acid/chaotropic treatment occurs concomitantly to the distribution of the complex into two distinct populations comprising $\mathrm{BChl}$ absorbing at $858 \mathrm{~nm}$ and BPheo absorbing at $843 \mathrm{~nm}$ respectively. It is demonstrated here that after acid/chaotropic treatment and reversion to standard conditions of buffer about $30-35 \%$ of the BChls are transformed into BPheo. Knowing the B800 BChl/ B850 BChl ratio (1:2) (McDermott et al., 1995; Koepke et al., 1996) and by virtue of previous spectral analysis of the reorganized LHII complex (Buche et al., 2000) it could be conclude that the transformation of $1 / 3$ of the BChls into BPheo induces the formation of the $843-\mathrm{nm}$ band while an other $1 / 3$ fraction constitutes the $858-\mathrm{nm}$ band. The remaining BChls are from B800, in dimeric or monomerised form (Buche et al., 2000). We proposed that the BPheos were responsible of the strong circular dichroism signal while B858 was assumed to arise from B850 BChls exciton interactions (Buche et al., 2000). If the heterogeneity of the reorganized $\mathrm{B} 850$ is a result of two distinct complexes the electronic interactions between the pigments must be radically different (Somsen et al., 1995; Koolhaas et al., 1997). In this case, it could be expected to separate these two complexes using an ionic liquid chromatography column.

However, the elution profile of a sample previously fixed, treated by acid/chaotropic conditions and reverted to standard conditions of buffer and $\mathrm{pH}$ reveals a great uniformity of B850. This result suggests that the $\mathrm{B} 850$ heterogeneity of the reorganized complex is not the effect of a distribution of the pigments into two different complexes. Alternatively, we propose that 843 - and $858-\mathrm{nm}$ bands are related to BChls fixed to two slightly different binding sites of the polypeptide.

\section{Acknowledgements}

We would like to thank María V. Ramiro for skilful technical assistance. The work was supported by the EC contract CT93-0278 and the DGICYT (Grant PB98-1632). 
Buche A. and Picorel R. (1998), Spectral changes induced by alkaline $\mathrm{pH}$ and specific chemical modification of amino acid residues in the light-harvesting II antenna complex from Ectothiorhodospira sp. Photochem. Photobiol. 69, 275-281.

Buche A., Ramirez J. M. and Picorel R. (2000), Effects of acid $\mathrm{pH}$ and urea on the spectral properties of the LHII antenna complex from the photosynthetic bacterium. Ectothiorhodospira sp. Eur. J. Biochem. 267, 3235-3243.

Buche A. and Picorel R. (2000), Absorption properties of the carotenoids after alkaline denaturation of the light-harvesting complex II from Ectothiorhodospira sp. Z. Naturforsch. 55c, 576-581.

Buche A. (2000), On the reorganisation of the LHII $850 \mathrm{~nm}$ band of Ectothiorhodospira sp. after acidic/ chaotropic treatment. J. Biochem. Mol. Biol. Biophys., (in press).

Clayton R. K. and Clayton B. J. (1981), B850 pigmentprotein complex of Rhodopseudomonas sphaeroides extinction coefficients, circular dichroism, and the reversible binding of bacteriochlorophyll. Proc. Natl. Acad. Sci. U. S. A. 78, 5583-5587.

Cogdell R. J., Durant, Y., Valentine Y., Lindsay J. G. and Schmidt K. (1983), The isolation and partial characterization of the light-harvesting pigment-protein complement of Rhodopseudomonas sphaeroides. Biochim. Biophys. Acta 722, 427-435.

Cogdell R. J. (1986), Light-harvesting complexes in the purple bacteria. In: Encyclopaedia of Plant Physiology, Photosynthesis, III vol. 19 (Staehelin, L. A. and Arntzen, C. J.,eds). Springer Publ., Berlin, pp. 252-259.

Doi M., Shioi, Y. Gad'on, N., Golecki J. R. and Drews G. ( 1991), Spectroscopical studies on the light-harvesting pigment-protein complex II from dark-aerobic grown cells of Rhodobacter sulfidophilus. Biochim. Biophys. Acta 1058, 235-241.

McDermott G., Prince S. M., Freer A. A., Hawthornthwaite-Lawless A. M., Papiz M. Z. , Cogdell R. J. and Isaacs N. W. (1995), Crystal structure of an integral membrane light-harvesting complex from photosynthetic bacteria. Nature 374, 517-521.

Feick R. and Drews G. (1978), Isolation and characterization of light-harvesting bacteriochlorophyll-protein complexes from Rhodopseudomonas capsulata. Biochim. Biophys. Acta 501, 499-513.

Hayashi H. and Morita S. (1980), Near-infrared absorption spectra of light-harvesting bacteriochlorophyll protein complexes from Chromatium vinosum. J. Biochem. (Tokyo) 88, 1251-1258.
Koepke J., Hu X., Muenke C., Schulten K. and Michel H. (1996), The crystal structure of the light-harvesting complex II (B800-850) from Rhodospirillum molischianum. Structure 4, 581-597.

Koolhaas M. H. C., van der Zwan G., van Mourik F. and van Grondelle R. (1997), Spectroscopy and structure of bacteriochlorophyll dimers. I. Structural consequences of nonconservative circular dichroism spectra. Biophys. J. 72, 1828-1841.

Lefebvre S., Picorel R., Cloutier Y. and Gingras G. (1984), Photoreaction center of Ectothiorhodospira sp. Pigment, heme, quinone, and polypeptide composition. Biochemistry 23, 5279-5287.

Monger T. G. and Parson W. W. (1977), Singlet-triplet fusion in Rhodopseudomonas sphaeroides chromatophores. A probe of the organization of the photosynthetic apparatus. Biochim. Biophys. Acta 460, 393-407.

Ortiz de Zarate I. and Picorel R. (1994), Spectral changes of the B800-B850 antenna complex from $E c$ tothiorhodospira $s p$. induced by detergent and salt treatment. Photosynth. Res. 41, 339-347.

Picorel R. and Gingras G. (1988), Preparative isolation and characterization of the B875 complex from Rhodobacter sphaeroides 2.4.1. Biochem. Cell Biol. 66, $442-448$.

Somsen O. J. G., van Grondelle R. and van Amerongen H. (1995), Spectral moments facilitate the analysis of circular dichroism spectra. Application to chlorosomes from Chloroflexus aurantiacus. In: Photosynthesis: from Light to Biosphere (Mathis, P., ed.). Kluwer Academic Publishers, Dordrecht, The Netherlands, Vol. I. pp. 263-266.

Sundström V. and van Grondelle R. (1996), Kinetics of excitation transfer and trapping in purple bacteria. In: Anoxygenic Photosynthetic Bacteria (Blankenship, R., Madigan, M. T. and Bauer, C. E., ed.). Kluwer Academic Publishers, Dordrecht, The Netherlands, pp. 349-372.

Walker T., Gad'on N., Steck K., Welte W. and Drews G. (1988), Isolation of reaction center and antenna complexes from the halophilic purple bacterium Rhodospirillum salexigens. Biochim. Biophys. Acta 933, 299-305. 